\title{
Novel Photocatalysts for the Decomposition of Organic Dyes \\ Based on Metal Organic Framework Compounds (MOFs)
}

\author{
Partha Mahata, ${ }^{1}$ Madras Giridhar ${ }^{2}$ and Srinivasan Natarajan ${ }^{1 *}$
}

\section{ELECTRONIC SUPPLEMENTARY INFORMATION}

\footnotetext{
*Corresponding Authors, E-mail: snatarajan@ sscu.iisc.ernet.in; giridhar@chemeng.iisc.ernet.in
} 


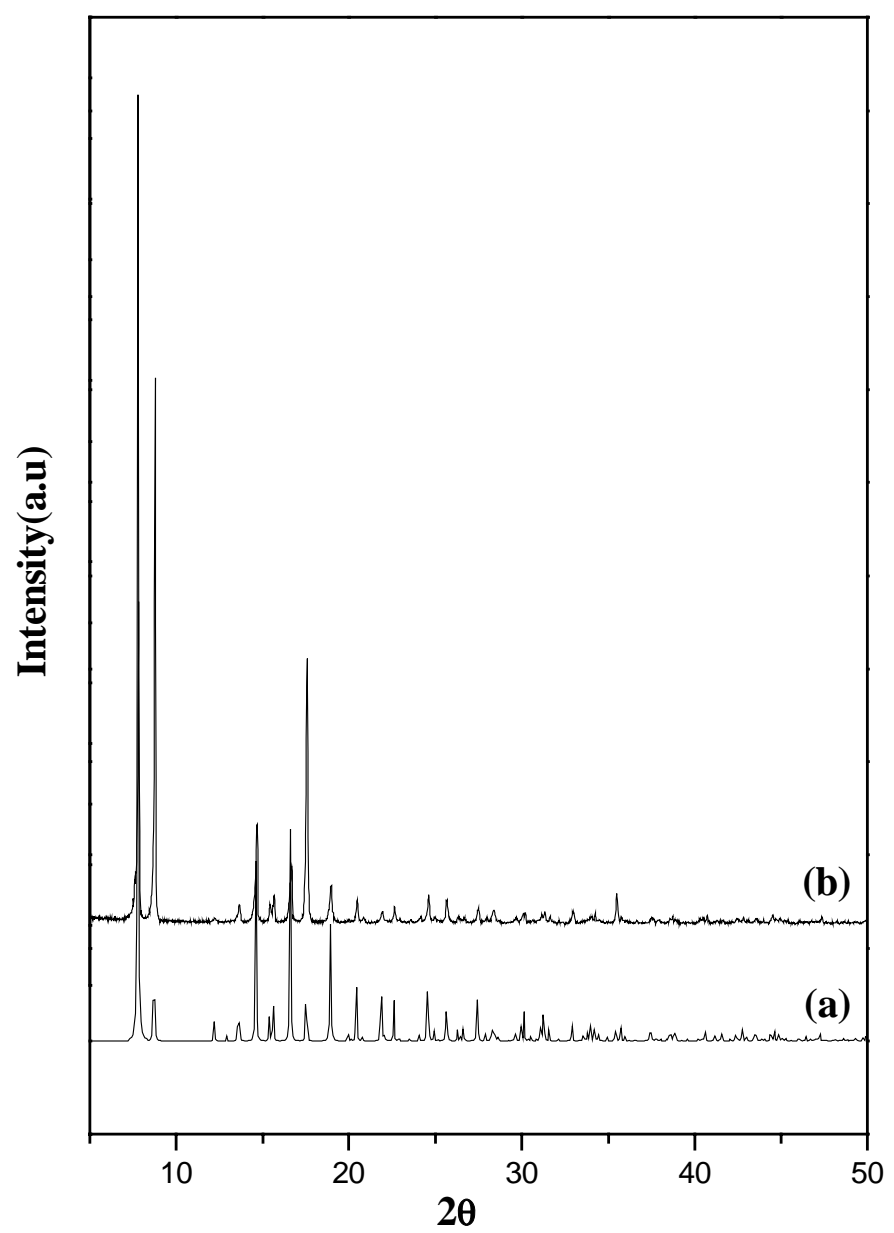

Fig. S1. Powder XRD (CuK $\alpha)$ pattern of 1: (a) simulated and (b) experimental 


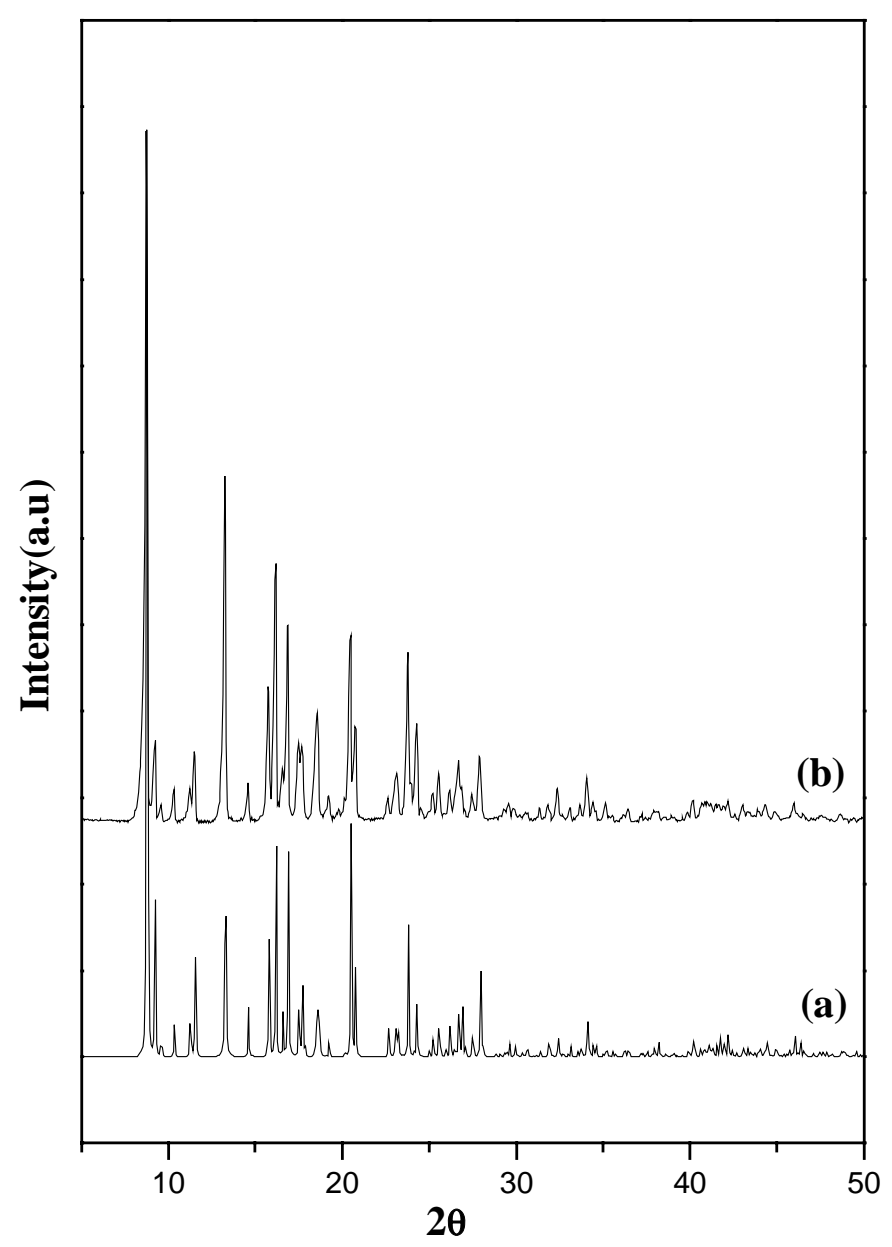

Fig. S2. Powder XRD (CuKo) pattern of 2: (a) simulated and (b) experimental 


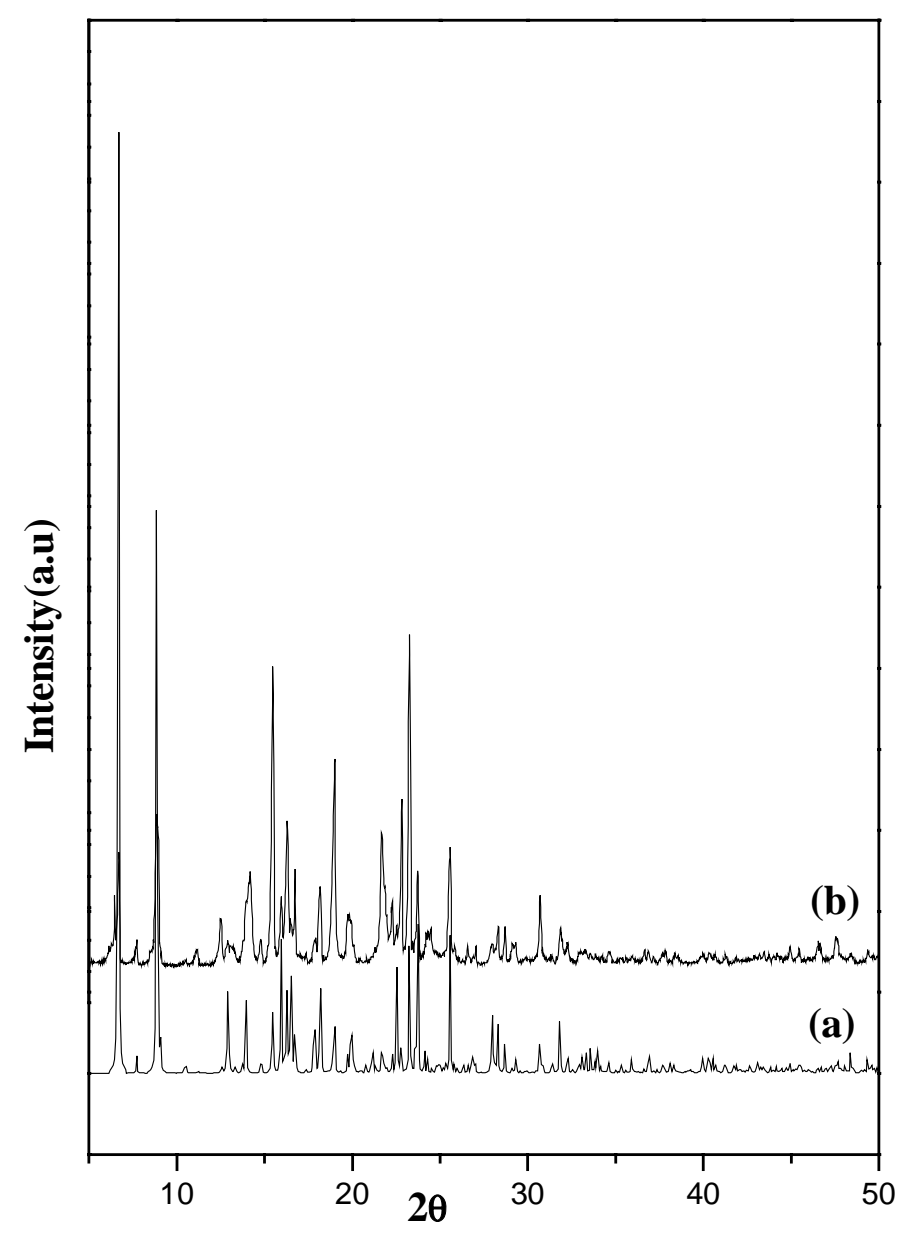

Fig. S3. Powder XRD (CuK $\alpha$ ) pattern of 3: (a) simulated and (b) experimental 


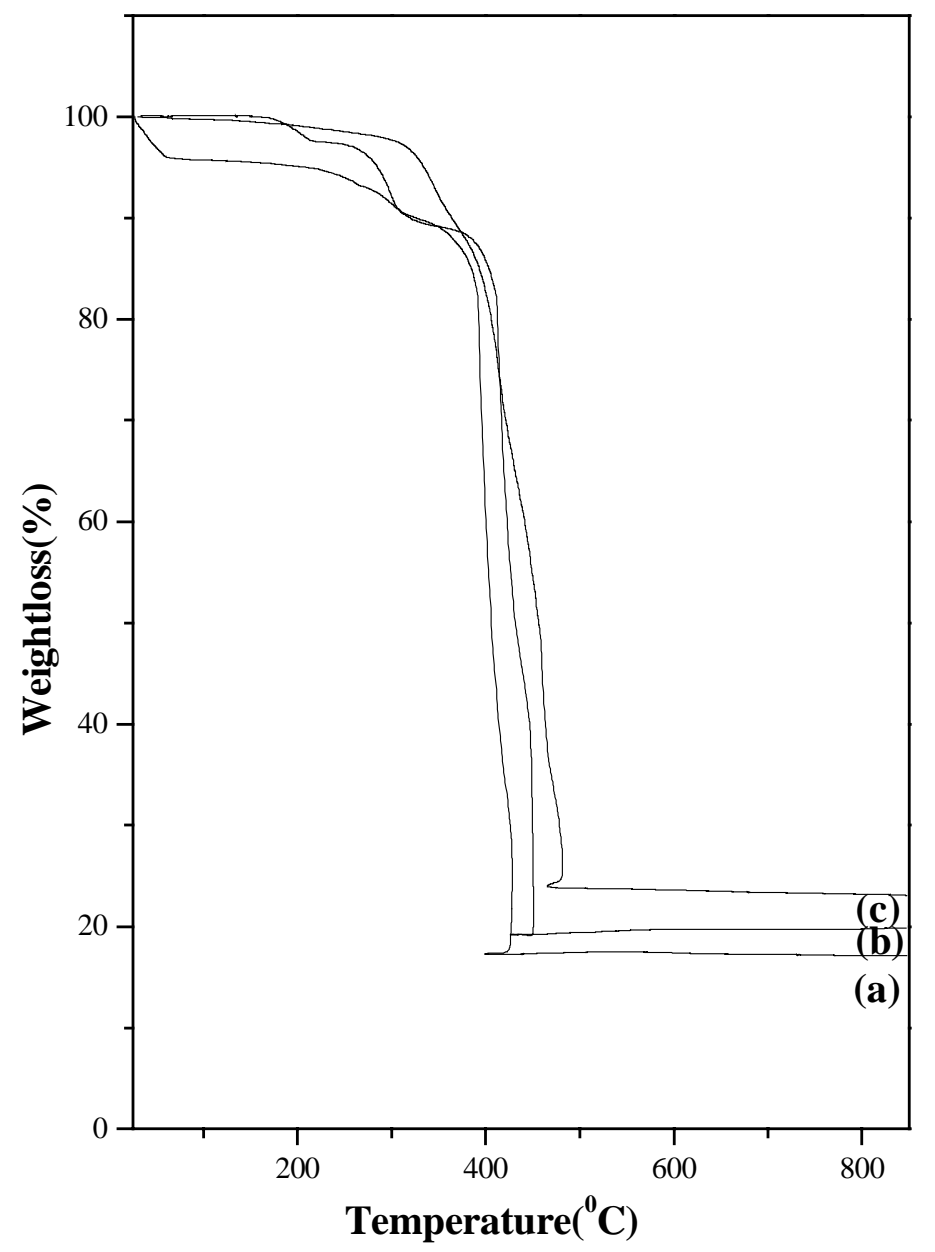

Fig. S4. TGA Studies of (a) 2, (b) 1, and (c) 3 


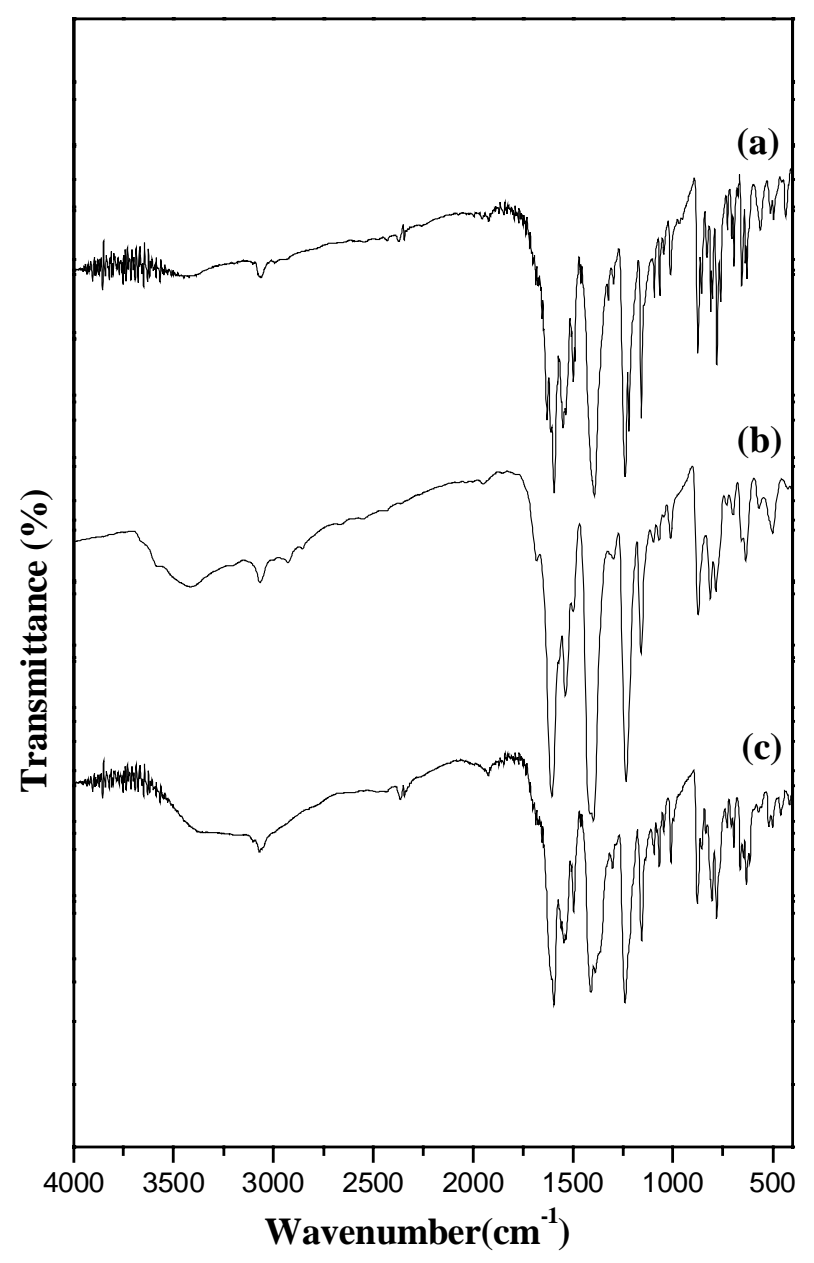

Fig. S5. IR spectra of (a) 1, (b) 2 and (c) 3 


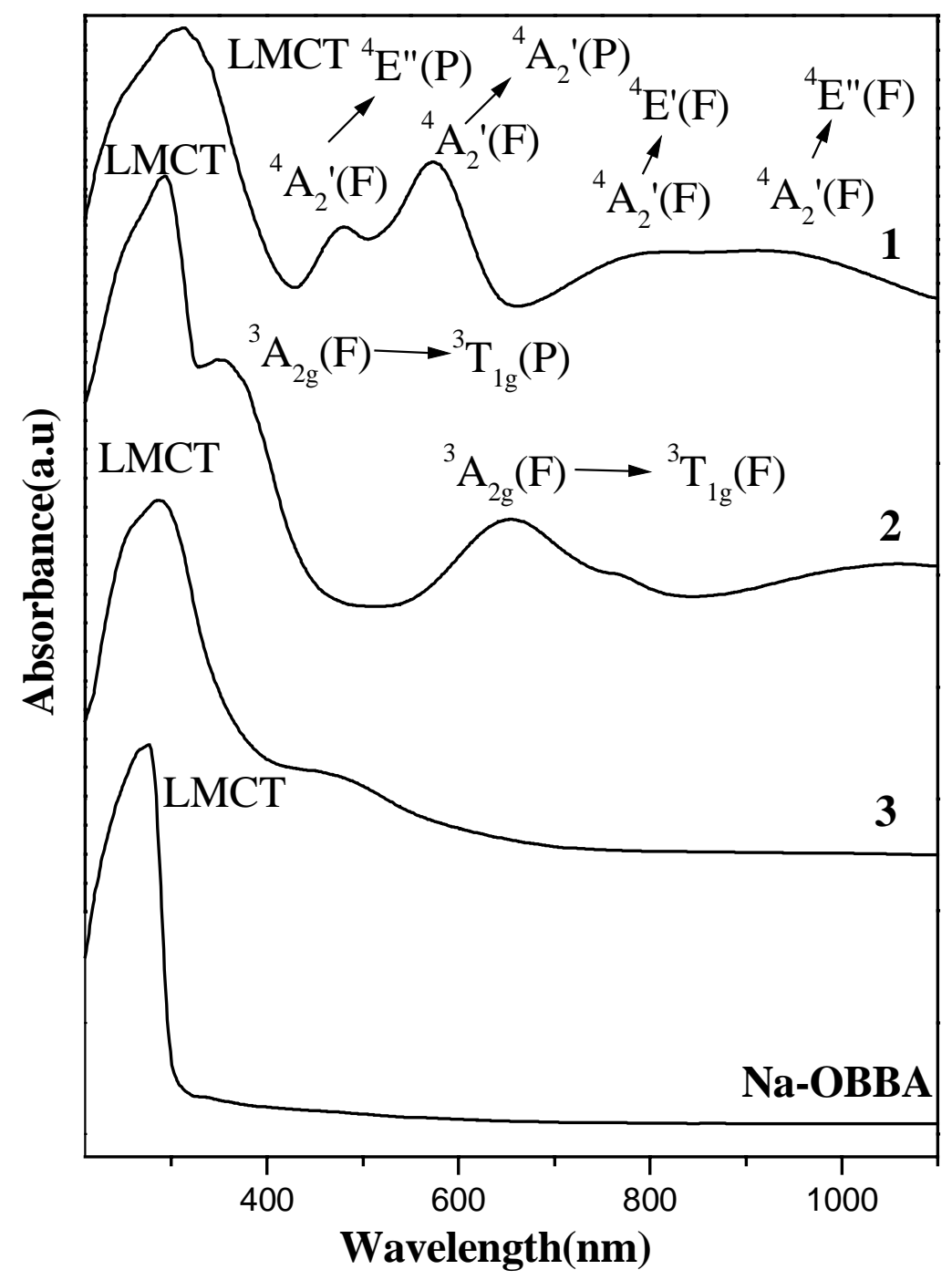

Fig. S6. UV - Visible spectra of the three compounds and Na salt of OBA 


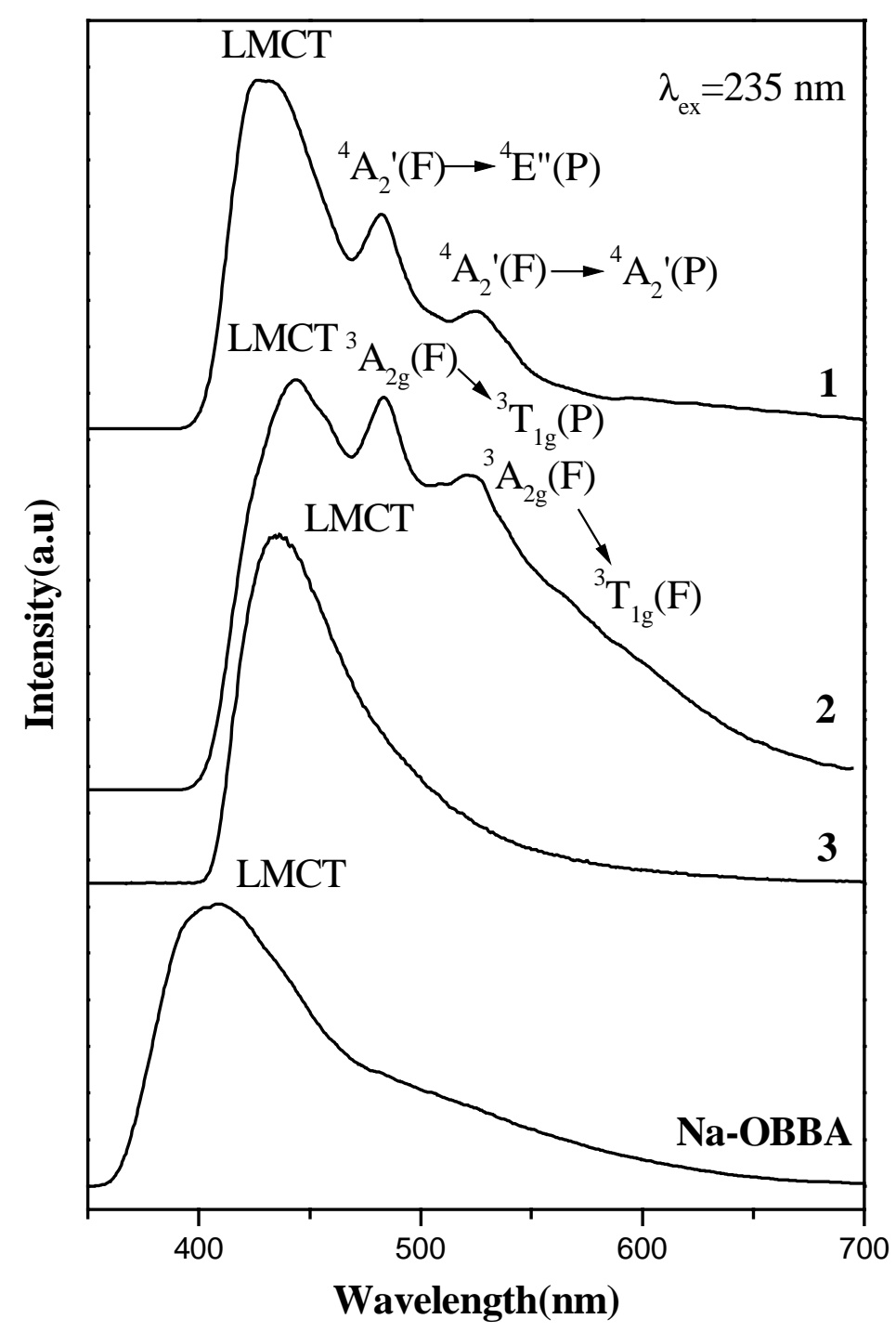

Fig. S7. Photoluminescence spectra of the three compounds and Na salt of OBA 
Table S1. Selected bond angles in $\mathbf{1},\left\{\left[\mathrm{Co}_{2}\left(\mathrm{C}_{10} \mathrm{H}_{8} \mathrm{~N}_{2}\right)\right]\left[\mathrm{C}_{12} \mathrm{H}_{8} \mathrm{O}(\mathrm{COO})_{2}\right]_{2}\right\}$

\begin{tabular}{llll}
\hline Angle & Amplitude $\left(^{\circ}\right)$ & Angle & Amplitude, $\left(^{\circ}\right)$ \\
\hline $\mathrm{O}(4)-\mathrm{Co}(1)-\mathrm{O}(1)$ & $112.17(12)$ & $\mathrm{O}(3)-\mathrm{Co}(1)-\mathrm{N}(1)$ & $88.86(12)$ \\
$\mathrm{O}(4)-\mathrm{Co}(1)-\mathrm{O}(3)$ & $106.48(11)$ & $\mathrm{O}(2)-\mathrm{Co}(1)-\mathrm{N}(1)$ & $171.21(12)$ \\
$\mathrm{O}(1)-\mathrm{Co}(1)-\mathrm{O}(3)$ & $141.00(12)$ & $\mathrm{C}(14) \# 1-\mathrm{O}(1)-\mathrm{Co}(1)$ & $128.0(3)$ \\
$\mathrm{O}(4)-\mathrm{Co}(1)-\mathrm{O}(2)$ & $89.37(11)$ & $\mathrm{C}(14)-\mathrm{O}(2)-\mathrm{Co}(1)$ & $131.7(3)$ \\
$\mathrm{O}(1)-\mathrm{Co}(1)-\mathrm{O}(2)$ & $85.77(11)$ & $\mathrm{C}(1)-\mathrm{O}(3)-\mathrm{Co}(1)$ & $126.6(2)$ \\
$\mathrm{O}(3)-\mathrm{Co}(1)-\mathrm{O}(2)$ & $99.93(11)$ & $\mathrm{C}(1) \# 2-\mathrm{O}(4)-\mathrm{Co}(1)$ & $132.8(3)$ \\
$\mathrm{O}(4)-\mathrm{Co}(1)-\mathrm{N}(1)$ & $88.20(12)$ & $\mathrm{C}(21)-\mathrm{N}(1)-\mathrm{Co}(1)$ & $118.8(3)$ \\
$\mathrm{O}(1)-\mathrm{Co}(1)-\mathrm{N}(1)$ & $87.33(11)$ & $\mathrm{C}(25)-\mathrm{N}(1)-\mathrm{Co}(1)$ & $125.3(3)$ \\
\end{tabular}

$\overline{\text { Symmetry transformations used to generate equivalent atoms: \#1 -x, y, -z+1/2 \#2-x, -y,-z+1 }}$ 
Table S2. Selected bond angles in $2,\left\{\left[\mathrm{Ni}_{2}\left(\mathrm{C}_{10} \mathrm{H}_{8} \mathrm{~N}_{2}\right)_{2}\right]\left[\mathrm{C}_{12} \mathrm{H}_{8} \mathrm{O}(\mathrm{COO})_{2}\right]_{2} \cdot \mathrm{H}_{2} \mathrm{O}\right\}$

\begin{tabular}{|c|c|c|c|}
\hline Angle & Amplitude $\left(^{\circ}\right)$ & Angle & Amplitude, $\left(^{\circ}\right)$ \\
\hline $\mathrm{O}(3)-\mathrm{Ni}(1)-\mathrm{O}(4)$ & $105.06(13)$ & $\mathrm{O}(6) \# 2-\mathrm{Ni}(2)-\mathrm{O}(7)$ & $156.55(12)$ \\
\hline $\mathrm{O}(3)-\mathrm{Ni}(1)-\mathrm{N}(2)$ & $92.66(13)$ & $\mathrm{N}(21)-\mathrm{Ni}(2)-\mathrm{O}(7)$ & $87.10(13)$ \\
\hline $\mathrm{O}(4)-\mathrm{Ni}(1)-\mathrm{N}(2)$ & $93.82(13)$ & $\mathrm{N}(4)-\mathrm{Ni}(2)-\mathrm{O}(8)$ & $90.46(13)$ \\
\hline $\mathrm{O}(3)-\mathrm{Ni}(1)-\mathrm{N}(1)$ & $86.72(11)$ & $\mathrm{O}(8)-\mathrm{Ni}(2)-\mathrm{O}(7)$ & $61.92(12)$ \\
\hline $\mathrm{O}(4)-\mathrm{Ni}(1)-\mathrm{N}(1)$ & $88.78(11)$ & $\mathrm{O}(5) \# 1-\mathrm{Ni}(2)-\mathrm{C}(54)$ & $129.20(15)$ \\
\hline $\mathrm{N}(2)-\mathrm{Ni}(1)-\mathrm{N}(1)$ & $117.40(11)$ & $\mathrm{O}(6) \# 2-\mathrm{Ni}(2)-\mathrm{C}(54)$ & $125.43(14)$ \\
\hline $\mathrm{O}(3)-\mathrm{Ni}(1)-\mathrm{O}(1)$ & $159.80(12)$ & $\mathrm{N}(21)-\mathrm{Ni}(2)-\mathrm{C}(54)$ & $88.86(14)$ \\
\hline $\mathrm{O}(4)-\mathrm{Ni}(1)-\mathrm{O}(1)$ & $94.67(12)$ & $\mathrm{N}(4)-\mathrm{Ni}(2)-\mathrm{C}(54)$ & $89.49(14)$ \\
\hline $\mathrm{N}(2)-\mathrm{Ni}(1)-\mathrm{O}(2)$ & $90.31(13)$ & $\mathrm{O}(8)-\mathrm{Ni}(2)-\mathrm{C}(54)$ & $30.80(13)$ \\
\hline $\mathrm{N}(1)-\mathrm{Ni}(1)-\mathrm{O}(2)$ & $89.42(13)$ & $\mathrm{O}(7)-\mathrm{Ni}(2)-\mathrm{C}(54)$ & $31.13(13)$ \\
\hline $\mathrm{O}(3)-\mathrm{Ni}(1)-\mathrm{O}(2)$ & $98.25(12)$ & $\mathrm{C}(21)-\mathrm{O}(1)-\mathrm{Ni}(1)$ & $89.2(3)$ \\
\hline $\mathrm{O}(4)-\mathrm{Ni}(1)-\mathrm{O}(2)$ & $156.60(12)$ & $\mathrm{C}(21)-\mathrm{O}(2)-\mathrm{Ni}(1)$ & $88.5(3)$ \\
\hline $\mathrm{N}(2)-\mathrm{Ni}(1)-\mathrm{O}(2)$ & $87.06(13)$ & $\mathrm{C}(41)-\mathrm{O}(3)-\mathrm{Ni}(1)$ & $128.7(3)$ \\
\hline $\mathrm{N}(1)-\mathrm{Ni}(1)-\mathrm{O}(2)$ & $90.53(13)$ & $\mathrm{C}(41) \# 3-\mathrm{O}(4)-\mathrm{Ni}(1)$ & $142.9(3)$ \\
\hline $\mathrm{O}(1)-\mathrm{Ni}(1)-\mathrm{O}(2)$ & $61.94(12)$ & $\mathrm{C}(34)-\mathrm{O}(5)-\mathrm{Ni}(2) \# 4$ & 128.1(3) \\
\hline $\mathrm{N}(1)-\mathrm{Ni}(1)-\mathrm{C}(21)$ & $89.46(14)$ & $\mathrm{C}(5)-\mathrm{N}(1)-\mathrm{Ni}(1)$ & 122.1(3) \\
\hline $\mathrm{O}(1)-\mathrm{Ni}(1)-\mathrm{C}(21)$ & $30.94(13)$ & $\mathrm{C}(1)-\mathrm{N}(1)-\mathrm{Ni}(1)$ & $121.5(3)$ \\
\hline $\mathrm{O}(2)-\mathrm{Ni}(1)-\mathrm{C}(21)$ & $31.01(13)$ & $\mathrm{C}(8) \# 6-\mathrm{N}(2)-\mathrm{Ni}(1)$ & $122.1(3)$ \\
\hline $\mathrm{O}(5) \# 1-\mathrm{Ni}(2)-\mathrm{O}(6) \# 2$ & $105.15(12)$ & $\mathrm{C}(9) \# 6-\mathrm{N}(2)-\mathrm{Ni}(1)$ & $121.3(3)$ \\
\hline $\mathrm{O}(5) \# 1-\mathrm{Ni}(2)-\mathrm{N}(21)$ & $92.75(13)$ & $\mathrm{C}(15)-\mathrm{N}(4)-\mathrm{Ni}(2)$ & $121.5(3)$ \\
\hline $\mathrm{O}(6) \# 2-\mathrm{Ni}(2)-\mathrm{N}(21)$ & $93.59(13)$ & $\mathrm{C}(11)-\mathrm{N}(4)-\mathrm{Ni}(2)$ & $122.3(3)$ \\
\hline $\mathrm{O}(5) \# 1-\mathrm{Ni}(2)-\mathrm{N}(4)$ & $86.76(13)$ & $\mathrm{C}(19)-\mathrm{N}(21)-\mathrm{Ni}(2)$ & $122.3(3)$ \\
\hline $\mathrm{O}(6) \# 2-\mathrm{Ni}(2)-\mathrm{N}(4)$ & $88.98(13)$ & $\mathrm{C}(16)-\mathrm{N}(21)-\mathrm{Ni}(2)$ & 121.1(3) \\
\hline $\mathrm{N}(21)-\mathrm{Ni}(2)-\mathrm{N}(4)$ & $177.42(14)$ & $\mathrm{O}(1)-\mathrm{C}(21)-\mathrm{Ni}(1)$ & $59.9(2)$ \\
\hline $\mathrm{O}(5) \# 1-\mathrm{Ni}(2)-\mathrm{O}(8)$ & $159.73(12)$ & $\mathrm{O}(2)-\mathrm{C}(21)-\mathrm{Ni}(1)$ & $60.5(2)$ \\
\hline $\mathrm{O}(6) \# 2-\mathrm{Ni}(2)-\mathrm{O}(8)$ & $94.63(12)$ & $\mathrm{C}(22)-\mathrm{C}(21)-\mathrm{Ni}(1)$ & $178.0(3)$ \\
\hline $\mathrm{N}(21)-\mathrm{Ni}(2)-\mathrm{O}(8)$ & $90.31(13)$ & $\mathrm{O}(8)-\mathrm{C}(54)-\mathrm{Ni}(2)$ & $60.0(2)$ \\
\hline $\mathrm{N}(4)-\mathrm{Ni}(2)-\mathrm{O}(8)$ & $89.29(13)$ & $\mathrm{O}(7)-\mathrm{C}(54)-\mathrm{Ni}(2)$ & $60.6(2)$ \\
\hline $\mathrm{O}(5) \# 1-\mathrm{Ni}(2)-\mathrm{O}(7)$ & $98.22(12)$ & $\mathrm{C}(51)-\mathrm{C}(54)-\mathrm{Ni}(2)$ & 177.7(3) \\
\hline
\end{tabular}

Symmetry transformations used to generate equivalent atoms: $\# 1 \mathrm{x}+2, \mathrm{y}-2, \mathrm{z} \# 2-\mathrm{x}+1,-\mathrm{y}+1,-\mathrm{z}+1 \quad \# 3-\mathrm{x}+1$, $-y+1,-z+2 \# 4 x-2, y+2, z \# 6 x, y+1, z$ 
Table S3. Selected bond angles in $\mathbf{3},\left\{\left[\mathrm{Zn}_{2}\left(\mathrm{C}_{10} \mathrm{H}_{8} \mathrm{~N}_{2}\right)\right]\left[\mathrm{C}_{12} \mathrm{H}_{8} \mathrm{O}(\mathrm{COO})_{2}\right]_{2}\right\}$

\begin{tabular}{|c|c|c|c|}
\hline Angle & Amplitude $\left(^{\circ}\right)$ & Angle & Amplitude, $\left(^{\circ}\right)$ \\
\hline $\mathrm{O}(3)-\mathrm{Zn}(1)-\mathrm{O}(2)$ & $136.31(8)$ & $\mathrm{O}(7)-\mathrm{Zn}(2)-\mathrm{O}(4) \# 2$ & $103.48(12)$ \\
\hline $\mathrm{O}(3)-\mathrm{Zn}(1)-\mathrm{O}(1)$ & $93.59(8)$ & $\mathrm{O}(5)-\mathrm{Zn}(2)-\mathrm{O}(4) \# 2$ & $95.76(10)$ \\
\hline $\mathrm{O}(2)-\mathrm{Zn}(1)-\mathrm{O}(1)$ & $129.95(8)$ & $\mathrm{C}(14)-\mathrm{O}(1)-\mathrm{Zn}(1)$ & $174.0(2)$ \\
\hline $\mathrm{O}(3)-\mathrm{Zn}(1)-\mathrm{N}(2)$ & $91.89(8)$ & $\mathrm{C}(14) \# 3-\mathrm{O}(2)-\mathrm{Zn}(1)$ & $103.32(17)$ \\
\hline $\mathrm{O}(2)-\mathrm{Zn}(1)-\mathrm{N}(2)$ & $90.51(8)$ & $\mathrm{C}(21)-\mathrm{O}(3)-\mathrm{Zn}(1)$ & $112.98(17)$ \\
\hline $\mathrm{O}(1)-\mathrm{Zn}(1)-\mathrm{N}(2)$ & $91.63(8)$ & $\mathrm{C}(34)-\mathrm{O}(4)-\mathrm{Zn}(2) \# 2$ & $144.1(2)$ \\
\hline $\mathrm{O}(3)-\mathrm{Zn}(1)-\mathrm{N}(1)$ & $87.13(8)$ & $\mathrm{C}(21)-\mathrm{O}(5)-\mathrm{Zn}(2)$ & 133.91(19) \\
\hline $\mathrm{O}(2)-\mathrm{Zn}(1)-\mathrm{N}(1)$ & $89.41(11)$ & $\mathrm{C}(34)-\mathrm{O}(6)-\mathrm{Zn}(2) \# 4$ & $138.3(2)$ \\
\hline $\mathrm{O}(1)-\mathrm{Zn}(1)-\mathrm{N}(1)$ & $89.57(8)$ & $\mathrm{C}(1)-\mathrm{O}(7)-\mathrm{Zn}(2)$ & $109.0(2)$ \\
\hline $\mathrm{N}(2)-\mathrm{Zn}(1)-\mathrm{N}(1)$ & $178.49(9)$ & $\mathrm{C}(45)-\mathrm{N}(1)-\mathrm{Zn}(1)$ & $122.82(19)$ \\
\hline $\mathrm{O}(6) \# 1-\mathrm{Zn}(2)-\mathrm{O}(7)$ & $114.09(10)$ & $\mathrm{C}(41)-\mathrm{N}(1)-\mathrm{Zn}(1)$ & $120.69(19)$ \\
\hline $\mathrm{O}(6) \# 1-\mathrm{Zn}(2)-\mathrm{O}(5)$ & $106.27(9)$ & $\mathrm{C}(48) \# 7-\mathrm{N}(2)-\mathrm{Zn}(1)$ & $121.60(19)$ \\
\hline $\mathrm{O}(7)-\mathrm{Zn}(2)-\mathrm{O}(5)$ & $117.81(10)$ & $\mathrm{C}(49) \# 7-\mathrm{N}(2)-\mathrm{Zn}(1)$ & $122.00(19)$ \\
\hline $\mathrm{O}(6) \# 1-\mathrm{Zn}(2)-\mathrm{O}(4) \# 2$ & $118.61(10)$ & & \\
\hline
\end{tabular}

Symmetry transformations used to generate equivalent atoms: \#1 x, y, z-1 \#2-x,-y+2,-z+1 \#3-x,-y+1,-z $\# 4 \mathrm{x}, \mathrm{y}, \mathrm{z}+1 \quad \# 7 \mathrm{x}+1, \mathrm{y}, \mathrm{z}$ 